\title{
ANESTESIA EN CIRUGÍA VITREORRETINIANA UTILIZANDO UN CATÉTER RETROBULBAR
}

\section{ANESTHESIA FOR VITREORETINAL SURGERY USING A RETROBULBAR CATHETER TECHNIQUE}

\author{
FALCÓ-MOLMENEU E ${ }^{1}$, SORLÍ-CLEMENTE E ${ }^{2}$, RAMOS-MARTÍ F² ${ }^{2}$ CABRERA-PESET A ${ }^{2}$, \\ ANDRÉS-CALVO G ${ }^{2}$, PERIS-MOLÉS M ${ }^{1}$
}

\section{RESUMEN}

Objetivos: Evaluar el manejo del dolor peroperatorio en la cirugía vitreorretiniana, utilizando un cateter retrobulbar que permita reinyecciones de anestésicos locales.

Métodos: Fueron incluidos en el estudio 25 pacientes, sometidos a cirugía vitreorretiniana, utilizando un catéter flexible introducido en el espacio intraconal, a través de una aguja espinal 22 G $11 / 2$ de 40x $0,7 \mathrm{~mm}$, con la que depositamos previamente $3,5 \mathrm{ml}$ de ropivacaína $0,75 \%$ retrobulbares, dejando posteriormente el catéter fijado a piel. Ante la presencia de dolor (grado 3 o superior) se reinyectaron en el postoperatorio $2 \mathrm{ml}$ de ropivacaína $0,2 \%$, y 2 $\mathrm{ml}$ al $0,75 \%$ si el dolor acontecía intraoperatoriamente. El catéter se mantuvo entre 24 y 48 horas.

Resultados: Un paciente (4\%) manifestó moderado dolor intraoperatorio, que cedió tras la administración de $2 \mathrm{ml}$ de ropivacaína $0,75 \%$ a través del catéter, y no ocasionó interrupción de la cirugía. Tres pacientes $(12 \%)$ precisaron una reinyección postoperatoria de $2 \mathrm{ml}$ de ropivacaína $0,2 \%$ por dolor. Las reinyecciones aliviaron el dolor de forma inmediata (menos de $3 \mathrm{~min}$ ). No se precisaron segundas reinyecciones, ni se detectaron complicaciones derivadas de la técnica.

\section{ABSTRACT}

Objective: To evaluate peroperative pain management using a flexible spinal anesthesia catheter introduced into the retrobulbar space which allows injections of local anesthetics for vitreoretinal surgery.

Methods: Twenty-five patients who underwent vitreoretinal surgery receiving retrobulbar anesthesia with $3.5 \mathrm{ml}$ ropivacaine $0.75 \%$. After injection, a catheter with spinal needle $22 \mathrm{G}$ x $11 / 2(40 \times 0.7$ $\mathrm{mm}$ ) was introduced to the retrobulbar muscle cone. The needle was withdrawn and the catheter was fixed. When the patient started to feel pain (grade 3 or higher), $2 \mathrm{ml}$ ropivacaine $0.75 \%$ was administrated through the catheter during surgery or $2 \mathrm{ml} \mathrm{ropi-}$ vacaine $0.2 \%$ in the postoperative period. The catheter was removed 24-48h later.

Results: During surgery, 1 patient (4\%) received a re-injection of $2 \mathrm{ml}$ ropivacaine $0.75 \%$ because of pain. Three patients (12\%) experienced pain of grade 3 or higher in the postoperative period and needed re-injection of $2 \mathrm{ml}$ ropivacaine $0.2 \%$. Re-injections were an effective method to achieve analgesia. Adverse effects were not noticed.

Conclusions: The retrobulbar catheter technique is a procedure which allows multiple re-injections of

\footnotetext{
Recibido: 5/5/05. Aceptado: 19/2/07.

Hospital General de Castellón (España)

1 Licenciado en Medicina. Especialista en anestesiología.

2 Licenciado en Medicina. Especialista en oftalmología.

Correspondencia:

Paseo Ribalta, $18,1 .^{\circ} \mathrm{C}$

12004 Castellón

España

E-mail: chelo.quique@tiscali.es
} 
Conclusiones: La cateterización del espacio retrobulbar permite la administración fraccionada de anestésicos locales, aportando a la cirugía vitreorretiniana un adecuado soporte anestésico, así como un control del dolor postoperatorio de forma inmediata, eficaz y segura.

Palabras clave: Cirugía vitreorretiniana, cateter retrobulbar, ropivacaína, anestesia locorregional. local anesthetics; it provides adequate analgesia during surgery and rapid, effective and safe postoperative pain management (Arch Soc Esp Oftalmol 2007; 82: 147-152).

Key words: Vitreoretinal surgery, retrobulbar catheter, ropivacaine, regional anesthesia.

\section{INTRODUCCIÓN}

La anestesia locorregional se utiliza habitualmente en procesos quirúrgicos oftalmológicos, siendo la cirugía de la catarata la intervención más frecuente. Desde que Koller realizó la primera operación de catarata mediante instilación de cocaína, la anestesia en cirugía ocular ha pasado por distintas etapas y fue la anestesia general la técnica más empleada durante muchas décadas; desde hace unos 20 años la anestesia locorregional se ha impuesto; las características de la cirugía y sobre todo el tipo de pacientes, habitualmente de edad avanzada y con enfermedades asociadas, hacen aconsejable ajustar la anestesia al tipo de cirugía para minimizar riesgos y disminuir la morbilidad.

En este ámbito tiene su espacio la cirugía vitreorretiniana que se practica generalmente utilizando anestesia locorregional mediante inyección única retroperibulbar. La mezcla $(1,2)$ al $50 \%$ de bupivacaína $0,75 \%$ (Svedocain ${ }^{\circledR} 0,75 \%$. Laboratorios INIBSA, S.A. Lliça de Vall, Barcelona) con lidocaína $2 \%$ (Lidocaina braun ${ }^{\circledR}$ Laboratorio Braun Medical S.A. Melsungen, Alemania) es de uso frecuente, utilizando volúmenes de 5-7 ml. Esta técnica proporciona un adecuado soporte a la cirugía, que no suele exceder los $120 \mathrm{~min}$; sin embargo existen casos complejos cuya duración es imprevisible.

Jonas JB et al (3) han descrito la utilización de un catéter flexible retrobulbar, presentándose como una alternativa válida en estos casos, pues nos permite el control del dolor en todo el peroperatorio; así como evitar la anestesia general en pacientes de alto riesgo.

Mediante el presente estudio evaluamos prospectivamente una variante de la anestesia retrobulbar en cirugía ocular.

\section{SUJETOS, MATERIAL Y MÉTODOS}

Tras la aprobación del comité ético del hospital, 25 pacientes ASA II-III (10 hombres y 15 mujeres); se incluyeron en el estudio, la media de edad fue de 68,44 D.E. 7,83 años (media \pm desviación estandard); rango de 51 a 84 años.

Fueron excluidos del estudio aquellos pacientes en los que se preveía mala colaboración, los grandes miopes, con longitud axial del globo mayor a 26 $\mathrm{mm}$, incapacidad para mantenerse en decúbito supino al menos 2 horas y aquellos en los que existían contraindicaciones para la práctica de técnicas anestésicas locorregionales.

Tras monitorización y anestesia tópica conjuntival con lidocaína al $2 \%$, practicamos el abordaje del espacio retrobulbar. Utilizamos una aguja espinal 22 G $11 / 2$, de Becton Dickinson de dimensiones $0,7 \times 40 \mathrm{~mm}$, al no disponer de un equipo comercial para anestesia retrobulbar con catéter. Con el paciente colocado en decúbito supino y con la mirada en posición neutra, desinfectamos la superficie cutánea con povidona yodada, accediendo al espacio retrobulbar por la vía de Labath, donde tras aspiración suave que descarte la situación intravascular de la punta de la aguja depositamos 3,5 $\mathrm{ml}$ de ropivacaína $0,75 \%$ (Naropin ${ }^{\circledR}$ Laboratorios Astra Zeneca. Madrid), sin hialuronidasa, a través de la misma aguja y sin retirarla introducimos en el espacio retrobulbar la punta de un catéter espinal flexible y radioopaco $27 \mathrm{Gx} 90 \mathrm{~cm}$ de Pajunk, retiramos su fino fiador, llenamos el cateter con $1 \mathrm{ml}$ de ropivacaína $0,75 \%$ para vaciar el aire de su interior, utilizando una jeringa de $1 \mathrm{ml}$ debido a que la pequeña sección del catéter no permite mayores presiones; conectamos mediante un adaptador el catéter a un microfiltro de partículas de $0,2 \mathrm{~mm}$. El catéter lo fijamos a 
la piel del paciente usando apósitos adhesivos y dejando el filtro protegido y en un lugar accesible (hombro o parrilla costal), para poder reinyectar en caso necesario (fig. 1). Finalmente colocamos balón de Honan 10 min hasta $30-35 \mathrm{mmHg}$.

Se comprobó tras la cirugía, la localización de la punta del catéter mediante ecografía ocular en cuatro pacientes y TAC (Tomografía axial computerizada) utilizando contraste en un paciente, observándose el relleno progresivo retrobulbar en los distintos cortes (fig. 2).

Todos los pacientes recibieron un refuerzo peribulbar en el techo de la órbita (unión del tercio interno con los dos tercios externos), veintidós con $1,5 \mathrm{ml}$ de ropivacaína $0,75 \%$ mientras que en los tres casos restantes por sospecha de localización peribulbar del catéter, al no conseguir aquinesia del músculo recto interno, el refuerzo peribulbar en el techo de la órbita se practicó con un mezcla al 50\% de ropivacaína $0,75 \%$ y lidocaína al $2 \%$ con un volumen de $3 \mathrm{ml}$.

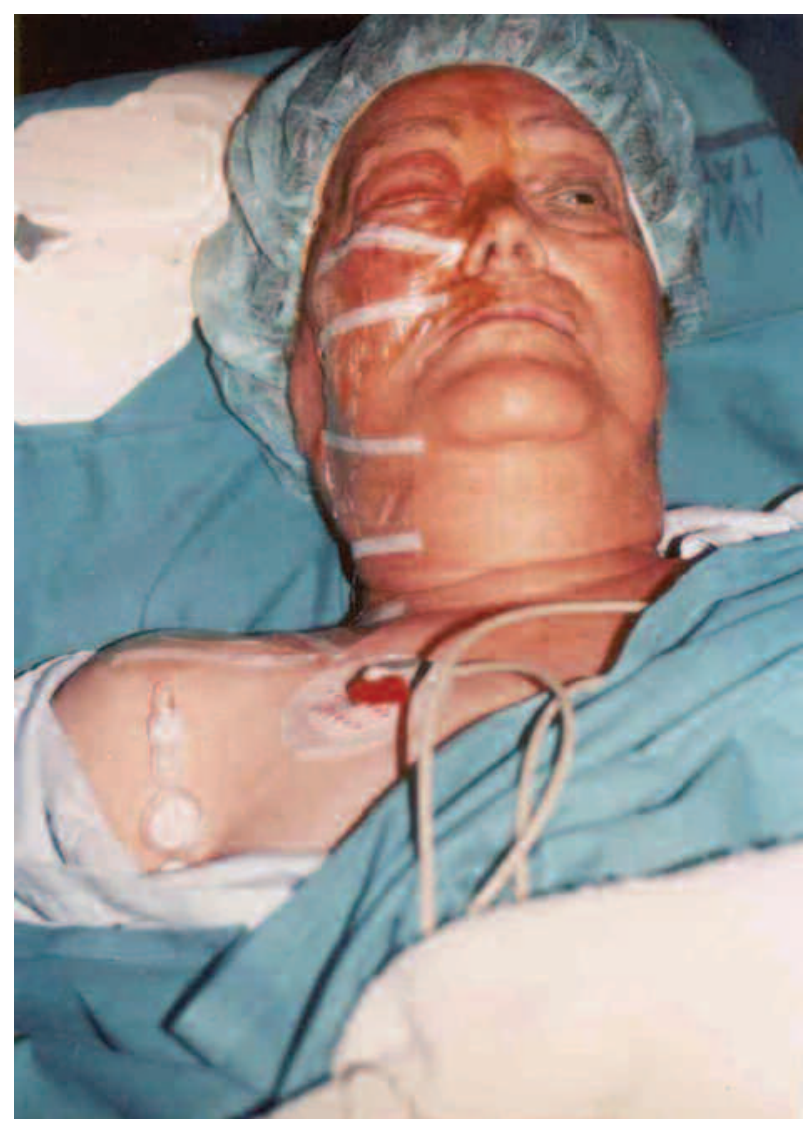

Fig. 1: Fijación del catéter a piel tras practicar bloqueo retrobulbar.

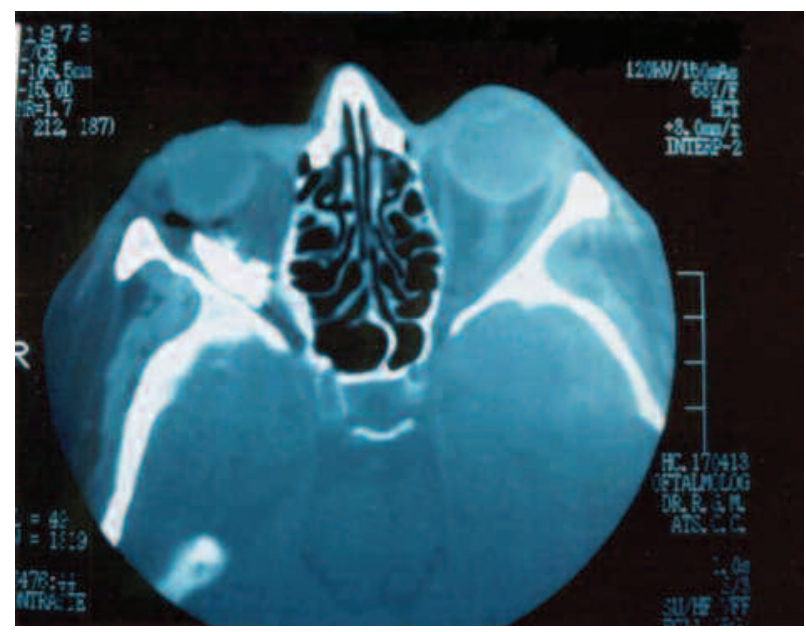

Fig. 2: TAC craneal donde se aprecia el relleno del espacio retrobulbar al administrar contraste a través del catéter.

Si durante el procedimiento quirúrgico el paciente sufría molestias o dolor se reinyectaban $2 \mathrm{ml}$ de ropivacaína $0,75 \%$ a través del catéter. Asimismo al finalizar la cirugía y estando los pacientes en la Unidad de Recuperación Postanestésica (URPA) o en la planta de hospitalización, eran interrogados a cerca de la comodidad de la técnica anestésica del grado de dolor que tenían según la Escala Analógica Visual (EAV) de modo que cuando referían un dolor superior a 3 en la EAV tras la cirugía, se procedía a reinyectar $2 \mathrm{ml}$ de ropivacaína al $0,2 \%$ a través del catéter.

Si transcurridas 24 horas tras la intervención quirúrgica el paciente no había experimentado dolor o éste era inferior a 3 en EAV el catéter era retirado, en caso contrario se mantenía hasta las 48 horas postcirugía.

\section{RESULTADOS}

La introducción del catéter retrobulbar a través de la aguja espinal no ocasionó molestias ni lesiones en el espacio retrobulbar, fue bien aceptado y tolerado por los pacientes y además de su utilidad quirúrgica también lo fue en el período postoperatorio para tratar el dolor agudo, mediante reinyecciones.

Precisaron reinyecciones cuatro pacientes (16\%), uno intraoperatoriamente y tres tras la intervención. Las intervenciones quirúrgicas tuvieron una dura- 
ción de 136 min D.E. 28,20 min (media \pm desviación estándar).

Las principales incidencias intraoperatorias fueron:

Un paciente, de los tres que precisaron refuerzo preoperatorio en el techo orbitario con $3 \mathrm{ml}$ por sospecha de localización peribulbar del catéter por mal bloqueo del músculo recto-interno y que el control ecográfico así lo sugirió, manifestó dolor durante la intervención quirúrgica transcurridos $95 \mathrm{~min}$ del bloqueo, que cedió en 3 min tras la administración de $2 \mathrm{ml}$ de ropivacaína $0,75 \%$ a través del catéter; eventualidad que no supuso una interrupción de la cirugía.

Dos pacientes $(8 \%)$ refirieron molestias en la apertura de la conjuntiva nasal (cuadrante superointerno), ambos habían presentado una aquinesia completa $($ Nicoll $=0)$ tras el bloqueo retrobulbar, que se resolvió mediante la instilación tópica de lidocaína $2 \%$ y no se reprodujeron ni dificultaron la cirugía posterior.

Tres pacientes $(12 \%)$ precisaron tratamiento iv por presentar hipertensión arterial intraoperatoria (todos ellos hipertensos conocidos).

Tras la cirugía y estando el paciente en planta de hospitalización:

Solo tres pacientes precisaron reinyecciones a través del catéter (12\%), uno trascurridos $270 \mathrm{~min}$. desde la realización del bloqueo retrobulbar, por presentar $\mathrm{EAV}=4$, un segundo a los $305 \mathrm{~min}$, por $\mathrm{EAV}=5$, y un tercero a los $312 \mathrm{~min}$ por $\mathrm{EAV}=4$, en todos ellos el alivio del dolor fue efectivo tras la reinyección de $2 \mathrm{ml}$ de ropivacaína $0,2 \%$, en un tiempo inferior a $3 \mathrm{~min}$, presentando posteriormente $\mathrm{EAV}=0-1$.

Un paciente refirió durante el período postoperatorio sensación de cuerpo extraño ocular, atribuible al roce del catéter con los tejidos circundantes, ya que se resolvió cuando a las 24 h fue retirado éste.

No se registraron casos de nauseas y vómitos peroperatorios, alteraciones de la motilidad ocular o infecciones, si bien nuestra serie ( 25 pacientes) es limitada.

Ningún paciente precisó una segunda reinyección.

El alivio inmediato del dolor fue valorado positivamente por todos los pacientes intervenidos mediante esta técnica anestésica, especialmente aquellos que habían experimentado otro tipo de anestesia para cirugía ocular y tratamiento del dolor postoperatorio.
En ningún caso fue necesario asociar analgésicos vía sistémica para controlar el dolor postoperatorio. No se detectaron efectos secundarios (hemodinámicos o centrales) derivados del uso de la ropivacaína retrobulbar. Asimismo no hubo infecciones locales o sistémicas, y el análisis bacteriológico de la punta de los 3 catéteres que se mantuvieron $48 \mathrm{~h}$ fue negativo.

Los pacientes valoraron el postoperatorio como bueno o excelente

\section{DISCUSIÓN}

La posibilidad de cateterizar el espacio retrobulbar (5) permite asistir a intervenciones quirúrgicas de larga duración en pacientes que podrían no verse beneficiados de la práctica de una anestesia general debido a su estado físico, garantizando el mantenimiento de la anestesia con la posibilidad de reinyecciones peroperatorias para el tratamiento del dolor agudo; a diferencia de las inyecciones únicas de anestésicos locales en los bloqueos que pierden efecto con el transcurso del tiempo, pudiendo provocar dolor e incomodidad al paciente, incluso durante la intervención quirúrgica.

En otras ocasiones la cirugía es limitada en el tiempo pero agresiva, con altos requerimientos en lo referente a la cobertura analgésica postoperatoria; los analgésicos sistémicos son útiles, pero no siempre lo eficaces que desearíamos en el alivio del dolor, ni su acción, tan inmediata como espera el paciente, sin olvidar que no están exentos, en ciertos pacientes susceptibles, de efectos no deseados.

Otra posibilidad en el tratamiento del dolor peroperatorio serían las reinyecciones de anestésicos locales, peri o retrobulbares, pero con potenciales riesgos (6) como la lesión muscular, vascular, nerviosa o la perforación del globo ocular (7) en una anatomía alterada después de la cirugía, además estas variantes no están exentas de críticas, ya que implican el manejo de volúmenes de anestésicos locales nada despreciables, que provocan endurecimiento y protusión del globo ocular no deseables.

El catéter retrobulbar puede ser una alternativa útil para las reinyecciones $(8,9)$ de anestésicos locales en el caso de optar por este tipo de anestesia y analgesia postoperatoria, que nos evita la introducción de nuevo de agujas en la órbita y permite el tratamiento del dolor agudo postoperatorio con pequeños volúmenes de anestésicos locales (10). 
El uso de ropivacaína, anestésico local tipo amida de acción larga con una toxicidad menor (11) si se compara con la bupivacaína racémica, aporta una analgesia prolongada del globo. Las dosis ajustadas a los requerimientos quirúrgicos y analgésicos utilizadas pueden considerarse bajas, lo cual unido a la menor toxicidad central (12) y cardiovascular (13) ya referida de este anestésico local frente a otros, nos aporta seguridad en su manejo.

El motivo por el cual reforzamos en todos los casos en el techo de la órbita con $1,5 \mathrm{ml}$ de ropivacaína $0,75 \%$ fue asegurar una correcta anestesia en el cuadrante supero-interno pues incluso habiendo sido apropiada la anestesia retrobulbar «ojo clavado», se observó eventualmente que la manipulación inicial en esta región fue dolorosa o al menos molesta a pesar de un correcto bloqueo.

La técnica anestésica retrobulbar la realizamos con el globo ocular en posición primaria o levemente hacia abajo y hacia fuera (posición de Unsöld), pues así la arteria oftálmica y sus ramas, la vena orbitaria superior y el nervio óptico se colocan más lejos de la punta de la aguja que si dirigimos el ojo hacia arriba y hacia dentro en el momento de la punción (posición de Atkinson).

La exploración practicada mediante TAC aportó datos fidedignos sobre la localización de la punta del catéter, sin embargo la ecografía ocular debido a la escasa «ventana» ofrecida por la anatomía de la cara (con muchos rebordes óseos) identifica con mayor dificultad la punta del catéter.

En la ecografía ocular practicada al paciente que precisó reinyección intraoperatoria a través del catéter, se observó una imagen sugestiva de localización peribulbar de la punta del mismo, lo cual es coincidente con un mal bloqueo del músculo recto interno observado tras la inyección (teniendo en cuenta la vía de abordaje inferior), que precisó antes del inicio de la intervención, un bloqueo suplementario en el techo de la órbita con $3 \mathrm{ml}(1,5 \mathrm{ml} \mathrm{de}$ lidocaina $2 \%+1,5 \mathrm{ml}$ de ropivacaína $0,75 \%$ ) comenzando ésta sin molestias hasta transcurridos 95 min. Este hecho y la bibliografía revisada $(14,15)$ nos sugiere la posibilidad de practicar la cirugía vitreorretiniana mediante cateterización del espacio peribulbar si bien precisaríamos con alta probabilidad de una inyección peribulbar única o doble para asegurar una correcta anestesia del globo, especialmente en el cuadrante superointerno, pero usando volúmenes mayores de anestésicos locales que en el caso de utilizar un catéter retro- bulbar. Sin embargo su aplicación y utilidad en la cirugía del polo anterior de larga duración o con un postoperatorio doloroso, como es el caso de cataratas complicadas, tumores de iris, y cirugía del trasplante de córnea, aportaría aspectos interesantes en el manejo anestésico de cierto tipo de pacientes, en los que la anestesia general pudiera aumentar la morbi-mortalidad de operaciones inicialmente poco agresivas pero laboriosas (16).

La no existencia de equipos comerciales retrobulbares (hasta hace poco tiempo) ha ocasionado que los materiales y accesos utilizados a lo largo de la historia por distintos profesionales para el abordaje del espacio retrobulbar hayan sido muy diversos, de este modo algunos autores con catéteres similares practican anestesias retrobulbares subtenonianas (17), mediante una pequeña disección de la conjuntiva, la cápsula de Tenon y el septo intermuscular, acceden al espacio retrobulbar por el cuadrante inferointerno o nasal de la órbita, donde depositan $3 \mathrm{ml}$ de anestésico local $(18,19)$; concluyen que se trata de una técnica sencilla, eficaz y segura, que evita la introducción de agujas en el interior de la órbita; si bien estos autores solo utilizan este procedimiento durante la cirugía (20) y no en el período postoperatorio.

Hay muchas variantes en técnicas locorregionales para cirugía ocular, como el uso de un catéter venoso (21) para abordar el espacio retrobulbar el cual puede fijarse y ser utilizado para reinyecciones postoperatorias. En la actualidad disponemos de un equipo específico para la órbita de polymedic ${ }^{\circledR}$ con el cual estamos trabajando.

Podemos concluir que mediante el catéter proporcionamos analgesia de forma inmediata y eficaz, administrando si fuera conveniente una última dosis de anestésico local, en nuestro caso $2 \mathrm{ml}$ de ropivacaína $0,2 \%$, antes de que el paciente fuera trasladado a planta. La ropivacaína aporta analgesia de larga duración, de tal modo que como ha ocurrido tras la cirugía, en 21 de los 25 pacientes intervenidos no fue necesaria ninguna reinyección, y en ningún caso fue necesario el uso de analgésicos y/o sedantes vía oral o parenteral.

Pensamos que el uso del catéter retrobulbar, que permita la administración de anestésicos locales en cirugía oftálmica, es un instrumento más del que disponen el anestesiólogo y el oftalmólogo para el manejo anestésico y analgésico de pacientes en los que queramos evitar una anestesia general y que permite ajustar con eficiencia la anestesia al grado de agresión quirúrgica. 


\section{BIBLIOGRAFÍA}

1. Benedetti S, Agostini A. Peribulbar anesthesia in vitreoretinal surgery. Retina 1994; 14: 277-280.

2. Gioia L, Prandi E, Codenotti M, Casati A, Fanelli G, Torri TM, et al. Peribulbar anestesia with either $0.75 \%$ ropivacaine or a $2 \%$ lidocaine and $0.5 \%$ bupivacaine mixture for vitreoretinal surgery: a double-blinded study. Anesth Analg 1999; 89: 739-742.

3. Jonas JB, Budde WM, Dinkel M, Hemmerling TM. Indwelling temporary retrobulbar catheter for long-lasting titratable local anesthesia. Arch Ophthalmol 2000; 118: 9961000.

4. Johnson RW. Anatomy for ophthalmic anaesthesia. Br J Anaesth 1995; 75: 80-87.

5. Laszlo CJ, Gombos K, Vimlati L, Salacz G, Hatvani I. A catheter technique in ophthalmic regional anaesthesia. Cadaver experiments. Acta Anaesthesiol Scand 2000; 44: 450-452.

6. Rubin AP. Complications of local anaesthesia for ophthalmic surgery. Br J Anaesth 1995; 75: 93-96.

7. Kimble JA, Morris RE, Witherspoon CD, Feist RM. Globe perforation from peribulbar injection. Arch Ophthalmol 1987; 105: 749.

8. Lincoff H, Kreissig I. A catheter to deliver retrobulbar medication. Arch Ophthalmol 1996; 114: 634-635.

9. Lincoff $H$, Kreissig I, Gelisken F, Stanga P. A retained catheter for retrobulbar administration of interferon for age-related macular degeneration. Dev Ophthalmol 1997; 29: 69-77.

10. Jonas JB, Hemmerling TM, Budde WM, Dinkel M. Postoperative analgesia by reinjections of local anesthetic through and indwelling retrobulbar catheter. Am J Ophthalmol 2000; 129: 54-58.

11. Feldman HS, Arthur GR, Covino BG. Comparative systemic toxicity of convulsant and supraconvulsant doses of intravenous ropivacaine, bupivacaine, and lidocaine in the conscious dog. Anesth Analg 1989; 69: 794-801.

12. Rutten AJ, Nancarrow C, Mather LE, Ilsley AH, Ruciman $W B$, Upton RN. Hemodynamic and central nervous system effect of intravenous bolus doses of lidocaine, bupivacaine, and ropivacaine in sheep. Anesth Analg 1989; 69: 291 299.

13. Moller R, Covino BG. Cardiac electrophysiologic properties of bupivacaine and lidocaine compared with those of ropivacaine, a new amide local anesthetic. Anesthesiology 1990; 72: 322-329.

14. Weiss JL, Deichman CB. A comparison of retrobulbar and periocular anesthesia for cataract surgery. Arch Ophthalmol 1989; 107: 96-98.

15. Bernard JM, Hommeril JL. Prolonged peribulbar anesthesia with indwelling catheter: a preliminary report of 217 cases. Br J Anaesth 1997; 78: 81-82.

16. Mein CE, Flynn HW Jr. Augmentation of local anesthesia during retinal detachment surgery. Arch Ophthalmol 1989; 107: 1084.

17. Bergman L, Berglin L, Algvere PV, Laurell CG, Stenkula S. Limbal sub-Tenon's administration of retrobulbar anesthesia using a blunt irrigating cannula. Ophthalmic Surg Lasers 1996; 27: 106-112.

18. Friedberg MA, Spellman FA, Pilkerton AR, Perrault LE Jr, Stephens RF. An alternative technique of local anesthesia for vitreoretinal surgery. Arch Ophthamol 1991; 109: 1615-1616.

19. Dantas PE, Nishiwaki-Dantas MC, Henriques MJ, de Almeida GV. Retrobulbar anesthesia with a flexible catheter. Ophthalmic Surg Lasers 1996; 27: 275-278.

20. Mein CE, Woodcock MG. Local anesthesia for vitreoretial surgery. Retina 1990; 10: 47-49.

21. Tamai M. A retained retrobulbar catheter for repetitive injection of anesthetics during ophthalmic surgery. Ophthalmic Surg 1983; 14: 579-581. 